Research Article

\title{
Inverse Projective Synchronization between Two Different Hyperchaotic Systems with Fractional Order
}

\author{
Yi Chai, ${ }^{1,2}$ Liping Chen, ${ }^{1,2}$ and Ranchao $\mathrm{Wu}^{3}$ \\ ${ }^{1}$ State Key Laboratory of Power Transmission Equipment \& System Security and New Technology, \\ Chongqing University, Chongqing 400030, China \\ 2 School of Automation, Chongqing University, Chongqing 400030, China \\ ${ }^{3}$ School of Mathematics, Anhui University, Hefei 230039, China \\ Correspondence should be addressed to Liping Chen, lip_chen@yahoo.com.cn
}

Received 14 July 2011; Accepted 5 October 2011

Academic Editor: Yuri Sotskov

Copyright (C) 2012 Yi Chai et al. This is an open access article distributed under the Creative Commons Attribution License, which permits unrestricted use, distribution, and reproduction in any medium, provided the original work is properly cited.

\begin{abstract}
This paper mainly investigates a novel inverse projective synchronization between two different fractional-order hyperchaotic systems, that is, the fractional-order hyperchaotic Lorenz system and the fractional-order hyperchaotic Chen system. By using the stability theory of fractionalorder differential equations and Lyapunov equations for fractional-order systems, two kinds of suitable controllers for achieving inverse projective synchronization are designed, in which the generalized synchronization, antisynchronization, and projective synchronization of fractionalorder hyperchaotic Lorenz system and fractional-order hyperchaotic Chen system are also successfully achieved, respectively. Finally, simulations are presented to demonstrate the validity and feasibility of the proposed method.
\end{abstract}

\section{Introduction}

The history of fractional calculus is more than three centuries old. It has been found that the behavior of many physical systems can be properly described by fractional-order systems, for example, dielectric polarization, electrode-electrolyte polarization, electromagnetic waves, viscoelastic systems, quantitative finance, and diffusion waves [1-3]. Compared with the classical integer-order models, fractional-order derivatives provide an excellent instrument for the description of memory and hereditary properties of various materials and processes. Nowadays, fractional-order systems have attracted more and more people's attention. Many authors began to investigate the chaotic dynamics of fractional-order nonlinear systems with the help of fractional calculus. For examples, Podlubny proposed a generalization of the PID controller for the control of the fractional-order systems [4], by using fractional calculus theory. Reference [5] investigated the chaos revealed by the pseudo inverse-based 
trajectory planning algorithms. With the introduction of fractional derivatives, it was proved that many fractional-order differential systems behave chaotically, such as, Chua's circuit of order as low as 2.7 can produce a chaotic attractor [6], chaos could exist in the fractionalorder Rössler equation with order as low as 2.4 [7]. In [8], the chaotic behavior and its control in the fractional-order Chen system is investigated. Sheu et al. studied the dynamics of the Newton-Leipnik system with fractional-order and found chaos existing in the fractionalorder system with order less than 3 [9]. Lü numerically investigate the chaotic behaviors of the fractional-order Lü system, in which the lowest order to have chaos is 0.3 [10]. It was shown that fractional-order modified Duffing system of order less than 2.2 can still behave in a chaotic manner [11]. A hyperchaotic attractor is characterized as a chaotic attractor with more than one positive Lyapunov exponent which can increase the randomness and higher unpredictability of the corresponding system. It is believed that chaotic systems with higher-dimensional attractors have much wider applications. Recently, some authors studied the fractional-order hyperchaotic system by adding nonlinear terms in fractionalorder systems. $\mathrm{Wu}$ and Liu found that hyperchaotic behavior does exist in the fractionalorder hyperchaotic Chen system of order as low as 3.72 through numerical simulations [12]. Reference [13] discussed numerically the lowest orders for hyperchaos in fractional-order hyperchaotic Lorenz system which is 3.884 . Hyperchaos can exist in the fractional-order Rössler hyperchaotic equation with order as low as 3.8 [14].

It has been well known that a chaotic system is a nonlinear deterministic system with unpredictable complexity. In the past decade, synchronization of chaotic systems has attracted considerable attention since the pioneering work of Pecora and Carroll [15]. The control and synchronization of chaotic systems have been developed and thoroughly studied over the past two decades for their applications in some engineering applications such as image processing, chemical and biological systems, information science, and secure communication [16, 17]. So far, many different types of synchronization have been introduced, such as complete synchronization [18], lag synchronization [19], generalized synchronization [20], impulsive synchronization [21], and phase synchronization [22]. In 2001, experimental investigations of antisynchronization of optically coupled semiconductor lasers with external cavities were presented by Wedekind and Parlitz [23]. Then Chil et al. [24] observed antisynchronization phenomena in coupled identical chaotic oscillators in 2003. Antisynchronization is a phenomenon that the state vectors of synchronized systems have the same amplitude but opposite signs as those of the driving system, which can be characterized by the vanishing of the sum of relevant variables. In the past ten years, various synchronization control laws are obtained by using different control methods, see [25-27]. In 1996, projective synchronization phenomenon was first reported and discussed by González-Miranda [28]. In 1999, Mainieri and Rehacek first proposed the concept of projective synchronization which is characterized by the fact that the drive and response systems could be synchronized up to a scaling factor [29]. Then, Li [30] considered a new synchronization method called modified projective synchronization (MPS), where the phases were locked and the amplitudes of the two coupled systems synchronized up to a scaling factor. The scaling factor is a constant transformation between the synchronized variables of the master and slave systems. In recent years, projective synchronization of chaotic systems has attracted increasing attention; some sufficient conditions for projective synchronization have been obtained, see [31-33].

Recently, synchronization of chaotic fractional differential systems becomes a challenging and interesting problem and starts to attract increasing attention due to its potential applications to secure communication and control processing. Most of the methods and results of chaos synchronization in the integer-order differential systems cannot be simply extended to the fractional-order systems because fractional differential dynamical systems 
are very complex. Some scholars did some research about projective synchronization of fractional systems. For instance, Shao et al. proposes a method to achieve projective synchronization of the fractional-order chaotic Rössler system [34]. Based on the idea of a nonlinear observer, Peng and Jiang proposed a new method and applied it to generalized projective synchronization for a class of fractional-order chaotic systems [35]; Wu and Wang discussed a new fractional-order system and investigated its projective synchronization by designing the suitable nonlinear controller [36]. The hybrid projective synchronization of different dimensional fractional-order chaotic systems with different dimensions is investigated in [37]. In the meantime, some scholars have obtained some results about antisynchronization of fractional-order chaotic system; for example, based on the idea of active control, a novel active pinning control strategy is presented to achieve the antisynchronization of new uncertain fractional-order unified chaotic systems in paper [38]. In fact, adopting higher-dimensional chaotic systems has been proposed for secure communication, and the presence of more than one Lyapunov exponent clearly improves the security of communication schemes by generating more complex dynamics. Interest in synchronization of the fractional-order hyperchaotic systems is again motivated by secure communication applications. References $[12,13,39]$ all discussed the synchronization of the fractional-order hyperchaotic systems. The antisynchronization of two identical and two nonidentical hyperchaotic fractional-order systems is investigated via sliding mode controller in [40], but the results about the projective synchronization and inverse synchronization are relatively few.

However, to our best knowledge, there are few results about inverse projective synchronization of fractional-order hyperchaotic system. Wu and $\mathrm{Lu}$ investigated the generalized projective synchronization of fractional-order Chen hyperchaotic systems [12]. The issue of full state hybrid projective synchronization of fractional Chen-Lee hyperchaotic system is investigated in [41]. They all concentrated on projective synchronization of fractional hyperchaotic systems, but without considering inverse synchronization. References $[38,40]$ only discussed inverse synchronization without considering projective synchronization. Motivated by the above discussions, in this letter, we study the inverse projective synchronization between fractional-order hyperchaotic Lorenz system and fractional-order hyperchaotic Chen system. Two novel control laws are derived for synchronizing fractionalorder hyperchaotic Lorenz system and fractional-order hyperchaotic Chen system.

The remainder of this paper is organized as follows. In Section 2, fractional-order hyperchaotic Lorenz system and fractional-order hyperchaotic Chen system are described, and preliminary results are presented. In Section 3, two control schemes of inverse projective synchronization for fractional-order hyperchaotic systems of different structures are presented. In Section 4, numerical simulations are given to illustrate the effectiveness of the main results. Finally, conclusions are drawn in Section 5.

\section{System Description and Preliminaries}

Fractional calculus is a generalization of integration and differentiation to a noninteger-order integrodifferential operator $D_{t}^{\alpha}$ defined by

$$
D_{t}^{\alpha}= \begin{cases}\frac{d^{\alpha}}{d t^{\alpha}}, & R(\alpha)>0 \\ 1, & R(\alpha)=0 \\ \int_{a}^{t}(d \tau)^{-\alpha}, & R(\alpha)<0 .\end{cases}
$$


There are some definitions for fractional derivatives. The commonly used definitions are Grunwald-Letnikov, Riemann-Liouville, and Caputo definitions. The Riemann-Liouvill fractional derivatives, defined by

$$
\frac{d^{\alpha} f(t)}{d t^{\alpha}}=J^{n-\alpha} \frac{d^{n} f(t)}{d t^{n}}
$$

where $n=[\alpha]$, that is, $n$ is the first integer which is not less than $\alpha, J^{\beta}$ is the $\beta$-order RiemannLiuvile integral operator which is described as follows:

$$
J^{\beta}=\frac{1}{\Gamma(\beta)} \int_{0}^{t} \frac{\varphi(\tau)}{(t-\tau)^{1-\beta}} d \tau
$$

where $\Gamma(\cdot)$ is the gamma function, $0<\beta \leq 1$.

In this paper, the following definition is used:

$$
D_{*}^{\alpha} x(t)=J^{n-\alpha} x^{(n)}(t),
$$

where $n=[\alpha]$, it is a common practice to call the operator $D_{*}^{\alpha}$ the Caputo differential operator of order $\alpha$ because it has apparently first been used for the solution of practical problems by Caputo.

To obtain our results, the following lemma is presented.

For a given autonomous linear system of fractional order

$$
D_{*}^{\alpha} x=A x,
$$

with $x(0)=x_{0}$, where $x \in R^{n}$ is the state vector, one has the following.

Lemma 2.1. The system (2.5) is

(i) asymptotically stable if and only if $\left|\arg \left(\lambda_{i}(A)\right)\right|>\alpha \pi / 2, i=1,2,3, \ldots$, where $\arg \left(\lambda_{i}(A)\right)$ denotes the argument of the eigenvalue $\lambda_{i}$ of $A$. In this case, the component of the state decay towards 0 like $t^{-\alpha}$;

(ii) stable if and only if either it is asymptotically stable or those critical eigenvalues which satisfy $\left|\arg \left(\lambda_{i}(A)\right)\right|=\alpha \pi / 2$ have geometric multiplicity one.

Figure 1 illustrates the stability region of the fractional-order system.

The hyperchaotic Lorenz system with fractional order [13] is described as follows:

$$
\begin{gathered}
D_{*}^{q} x_{1}=a\left(x_{2}-x_{1}\right)+x_{4}, \\
D_{*}^{q} x_{2}=c x_{1}-x_{2}-x_{1} x_{3}, \\
D_{*}^{q} x_{3}=x_{1} x_{2}-b x_{3}, \\
D_{*}^{q} x_{4}=-x_{2} x_{3}+d x_{4},
\end{gathered}
$$

where $q$ is the fractional order, $0<q<1, x_{1}, x_{2}, x_{3}$, and $x_{4}$ are state variables, $a, b, c$, and $d$ are parameters. When the parameters are chosen as $a=10, b=8 / 3, c=28, d=-1$, and $q=0.98$, 


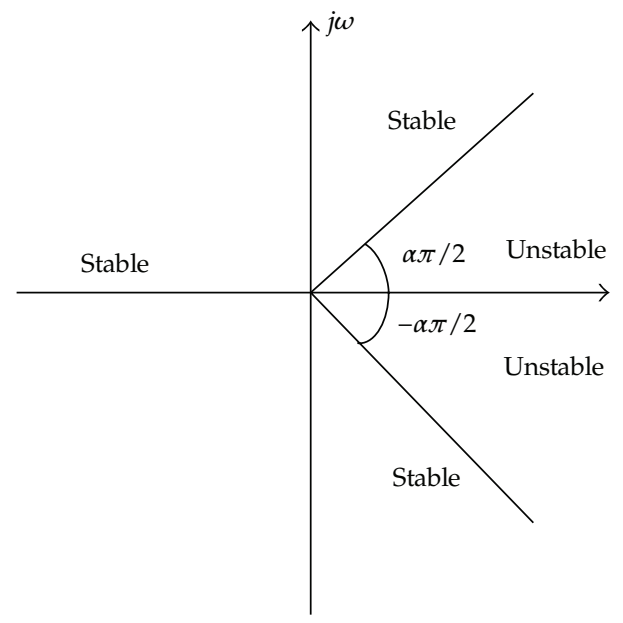

Figure 1: Stability region of the fractional-order system.

the values of Lyapunov exponents are $\lambda_{1}=0.3362, \lambda_{2}=0.1568, \lambda_{3}=0$, and $\lambda_{4}=-15.1724$, the system (2.6) exhibits hyperchaotic behaviors as shown in Figure 2. In 2009, Wu and Lu investigated the hyperchaotic behaviors in fractional-order Chen hyperchaotic system, which is described by

$$
\begin{gathered}
D_{*}^{q} y_{1}=\alpha\left(y_{2}-y_{1}\right)+y_{4}, \\
D_{*}^{q} y_{2}=\beta y_{1}-y_{1} y_{3}+\theta y_{2}, \\
D_{*}^{q} y_{3}=y_{1} y_{2}-r y_{3}, \\
D_{*}^{q} y_{4}=y_{2} y_{3}+\eta y_{4} .
\end{gathered}
$$

System (2.7) admits a hyperchaotic attractor for $\alpha=35, \beta=7, \theta=12, \gamma=3$, and $\eta=0.5$. Figure 3 displays the hyperchaotic attractor of fractional-order Chen system with fractionalorder $q=0.98$.

\section{Inverse Projective Synchronization}

In the section, we will discuss inverse projective synchronization behavior between fractional-order hyperchaotic Lorenz system and fractional-order hyperchaotic Chen system. We assume that fractional-order hyperchaotic Lorenz system is the drive system and the response system is fractional-order hyperchaotic Chen system, which are governed by systems (3.1) and (3.2), respectively,

$$
\begin{aligned}
& D_{*}^{q} x_{1}=a\left(x_{2}-x_{1}\right)+x_{4}, \\
& D_{*}^{q} x_{2}=c x_{1}-x_{2}-x_{1} x_{3}, \\
& D_{*}^{q} x_{3}=x_{1} x_{2}-b x_{3}, \\
& D_{*}^{q} x_{4}=-x_{2} x_{3}+d x_{4},
\end{aligned}
$$




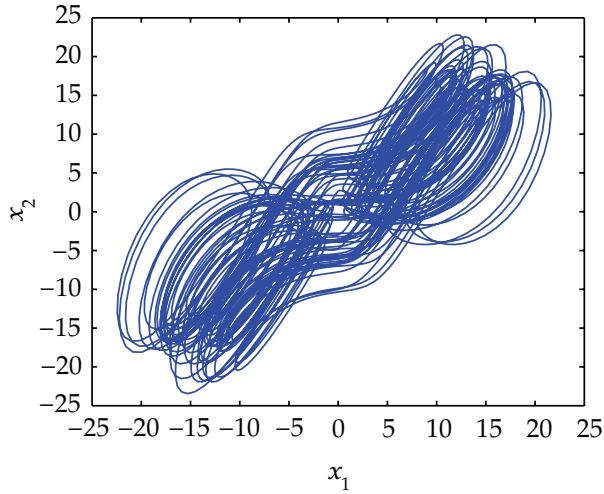

(a)

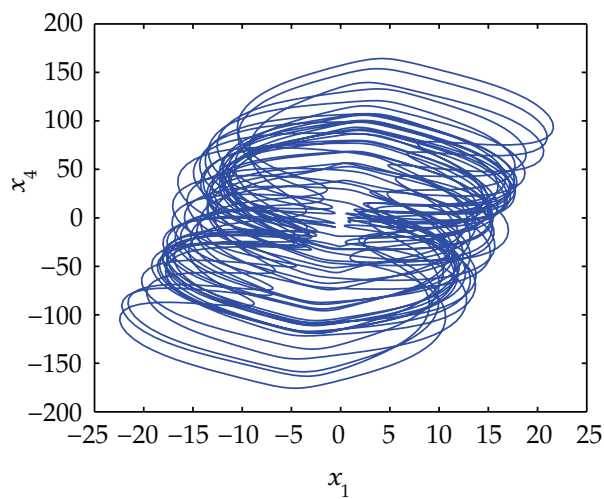

(c)

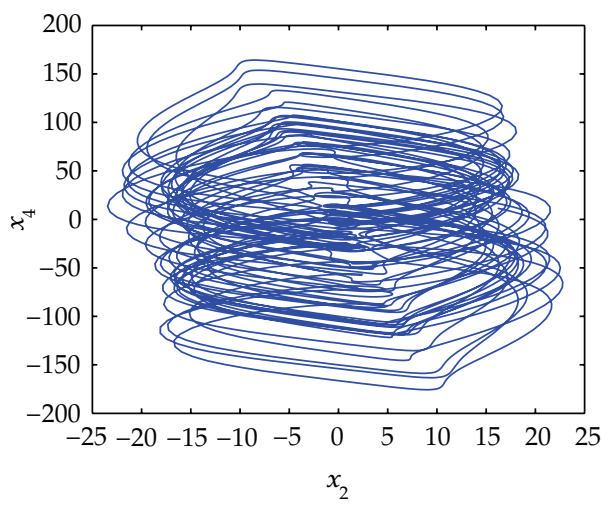

(e)

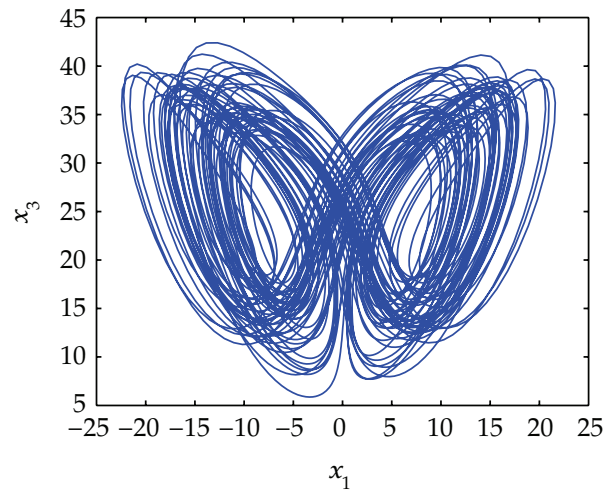

(b)

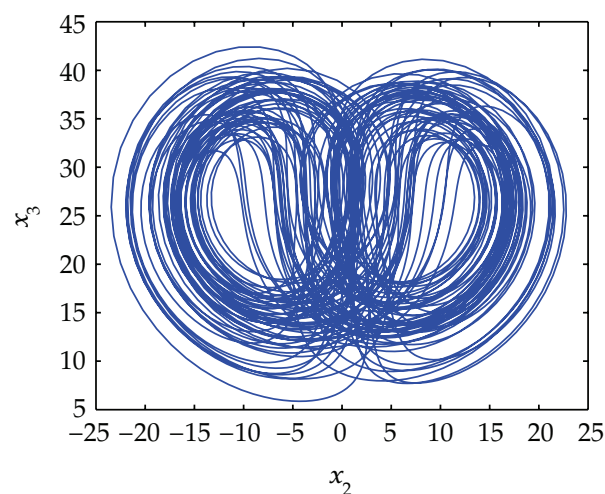

(d)

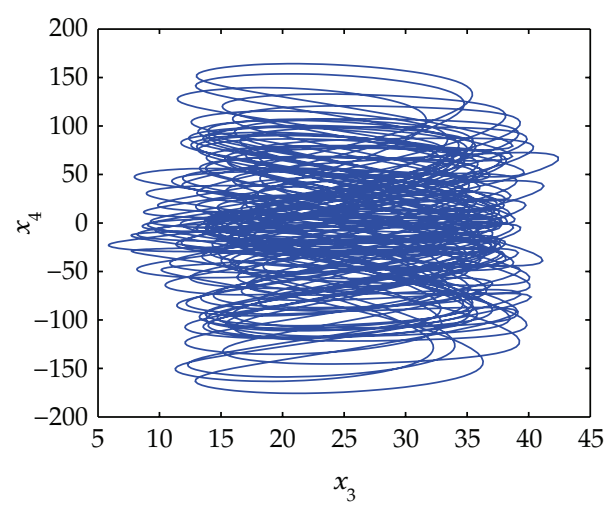

(f)

Figure 2: The projections of attractors of fractional-order hyperchaotic Lorenz system with order $q=0.98$.

$$
\begin{aligned}
& D_{*}^{q} y_{1}=\alpha\left(y_{2}-y_{1}\right)+y_{4}+u_{1}, \\
& D_{*}^{q} y_{2}=\beta y_{1}-y_{1} y_{3}+\theta y_{2}+u_{2}, \\
& D_{*}^{q} y_{3}=y_{1} y_{2}-r y_{3}+u_{3}, \\
& D_{*}^{q} y_{4}=y_{2} y_{3}+\eta y_{4}+u_{4},
\end{aligned}
$$


where $x_{i}$ and $y_{i}$ stand for state variables of the master system and the slave one, respectively. $a, b, c, d, \alpha, \beta, \theta, \gamma$, and $\eta$ are parameters. $u_{1}, u_{2}, u_{3}$, and $u_{4}$ are four nonlinear controllers will be designed later.

Definition 3.1. System (3.1) and (3.2) are inverse projective synchronization, if there exist a scaling matrix $\sigma$ such that

$$
\lim _{t \rightarrow \infty}\|e(t)\|=\lim _{t \rightarrow \infty}\|y+\sigma x\|=0,
$$

where $\|\cdot\|$ is the Euclidean norm and $\sigma=\operatorname{diag}\left(\sigma_{1}, \sigma_{2}, \sigma_{3}, \sigma_{4}\right)$.

It follows from (3.1)-(3.3) that we have the following error dynamical system:

$$
\begin{aligned}
& D_{*}^{q} e_{1}=\sigma y_{2}-\sigma y_{1}+y_{4}+a \sigma_{1} x_{2}-a \sigma_{1} x_{1}+\sigma_{1} x_{4}+u_{1}, \\
& D_{*}^{q} e_{2}=\beta y_{1}-y_{1} y_{3}+\theta y_{2}+c \sigma_{2} x_{1}-\sigma_{2} x_{2}-\sigma_{2} x_{1} x_{3}+u_{2}, \\
& D_{*}^{q} e_{3}=y_{1} y_{2}-\gamma y_{3}+\sigma_{3} x_{1} x_{2}-b \sigma_{3} x_{3}+u_{3} \\
& D_{*}^{q} e_{4}=y_{2} y_{3}+\eta y_{4}-\sigma_{4} x_{2} x_{3}+d \sigma_{4} x_{4}+u_{4}
\end{aligned}
$$

where $e_{1}=y_{1}+\sigma_{1} x_{1}, e_{2}=y_{2}+\sigma_{2} x_{2}, e_{3}=y_{3}+\sigma_{3} x_{3}, e_{4}=y_{4}+\sigma_{4} x_{4}$.

Our aim is to find suitable control laws $u_{i}(i=1,2,3,4)$ for stabilizing the error variables of the system at the origin. For this goal, we propose the following control laws for the error dynamical system

$$
\begin{aligned}
& u_{1}=(a-\alpha) \sigma_{1} x_{1}+\left(\alpha \sigma_{2}-a \sigma_{1}\right) x_{2}+\left(\sigma_{4}-\sigma_{1}\right) x_{4}-\left(\beta+\sigma_{3} x_{3}\right) e_{2}+\sigma_{2} x_{2} e_{3}, \\
& u_{2}=\left(\beta \sigma_{1}-c \sigma_{2}\right) x_{1}+(\theta+1) \sigma_{2} x_{2}+\left(\sigma_{1} \sigma_{3}+\sigma_{2}\right) x_{1} x_{3}+\left(e_{3}-\alpha\right) e_{1}-2 \theta e_{2}+\sigma_{3} x_{3} e_{4}, \\
& u_{3}=-\left(\sigma_{1} \sigma_{2}+\sigma_{3}\right) x_{1} x_{2}-(\gamma-b) \sigma_{3} x_{3}-e_{1} e_{2}+\sigma_{2} x_{2} e_{4} \\
& u_{4}=\left(\sigma_{4}-\sigma_{2} \sigma_{3}\right) x_{2} x_{3}+(\eta-d) \sigma_{4} x_{4}-e_{1}-e_{2} e_{3}-2 \eta e_{4} .
\end{aligned}
$$

Theorem 3.2. For a given constant scaling matrix $\sigma=\operatorname{diag}\left(\sigma_{1}, \sigma_{2}, \sigma_{3}, \sigma_{4}\right)$ and any initial conditions, if parameters $\alpha, \theta, \gamma$, and $\eta>0$, then the inverse projective synchronization between fractionalorder hyperchaotic Lorenz system (3.1) and Chen system (3.2) will occur under control law (3.5).

Proof. Substituting (3.5) into (3.4) leads to the following error system:

$$
\begin{aligned}
& D_{*}^{q} e_{1}=-\alpha e_{1}+\left(\alpha-\beta-\sigma_{3} x_{3}\right) e_{2}+\sigma_{2} x_{2} e_{3}+e_{4} \\
& D_{*}^{q} e_{2}=\left(\beta+\sigma_{3} x_{3}-\alpha\right) e_{1}-\theta e_{2}+\sigma_{1} x_{1} e_{3}+\sigma_{3} x_{3} e_{4} \\
& D_{*}^{q} e_{3}=-\sigma_{2} x_{2} e_{1}-\sigma_{1} x_{1} e_{2}-\gamma e_{3}+\sigma_{2} x_{2} e_{4} \\
& D_{*}^{q} e_{4}=-e_{1}-\sigma_{3} x_{3} e_{2}-\sigma_{2} x_{2} e_{3}-\eta e_{4} .
\end{aligned}
$$




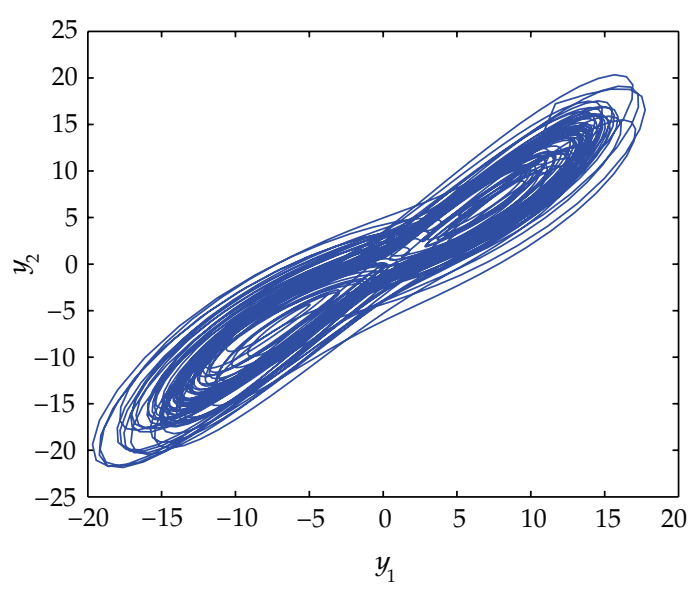

(a)

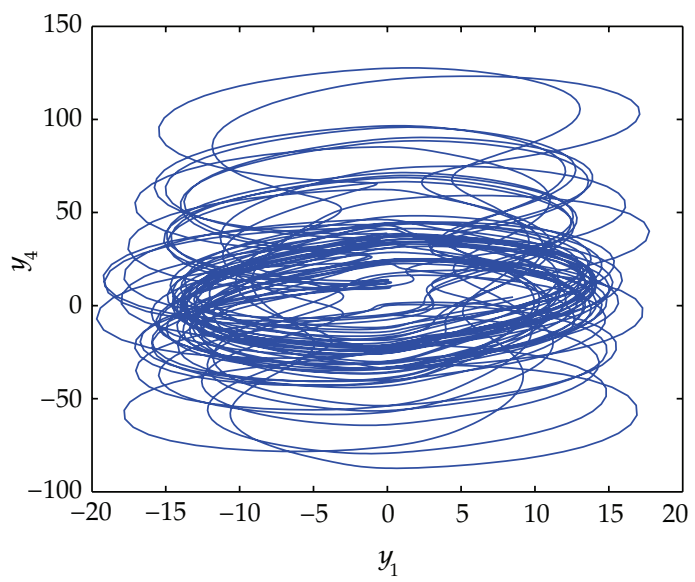

(c)

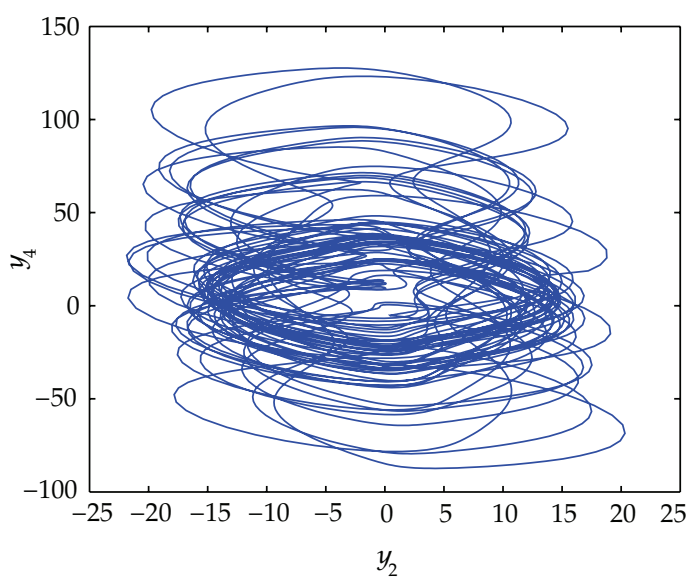

(e)

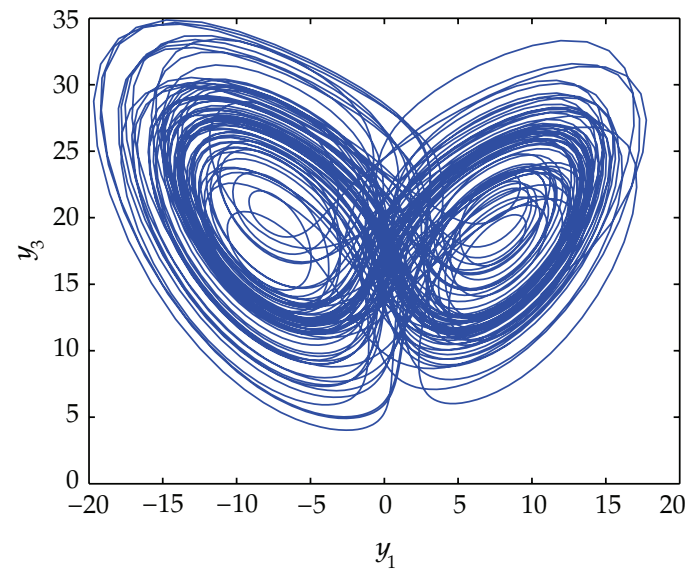

(b)

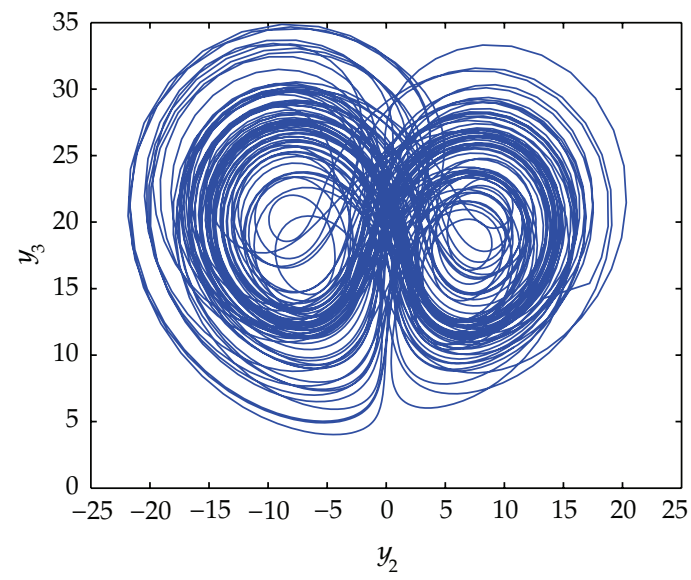

(d)

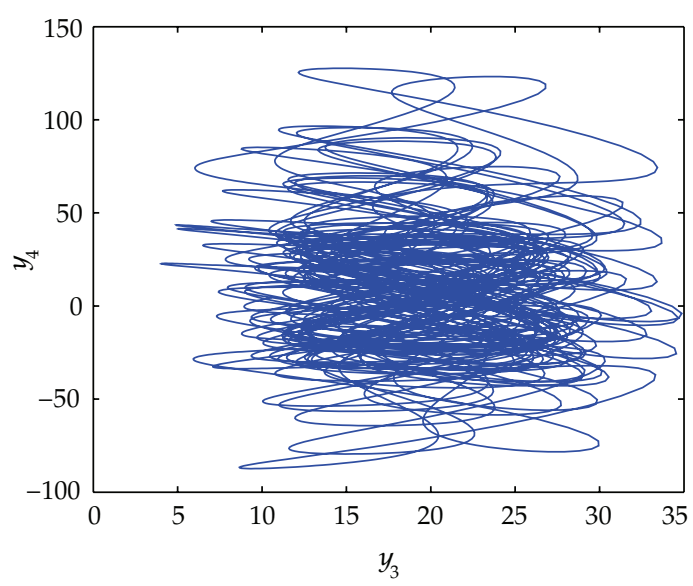

(f)

Figure 3: The projections of attractors of fractional-order hyperchaotic Chen system with order $q=0.98$. 
Error system (3.6) can be rewritten in the following matrix form:

$$
\left[D_{*}^{q} e_{1}, D_{*}^{q} e_{2}, D_{*}^{q} e_{3}, D_{*}^{q} e_{4}\right]^{T}=A\left[e_{1}, e_{2}, e_{3}, e_{4}\right]^{T}
$$

where

$$
A=\left[\begin{array}{cccc}
-\alpha & \alpha-\beta-\sigma_{3} x_{3} & \sigma_{2} x_{2} & 1 \\
\beta+\sigma_{3} x_{3}-\alpha & -\theta & \sigma_{1} x_{1} & \sigma_{3} x_{3} \\
-\sigma_{2} x_{2} & -\sigma_{1} x_{1} & -\gamma & \sigma_{2} x_{2} \\
-1 & -\sigma_{3} x_{3} & -\sigma_{2} x_{2} & -\eta
\end{array}\right]
$$

Suppose $\lambda$ is one of the eigenvalues of matrix $A$ and the corresponding eigenvector is $\varepsilon=\left(\varepsilon_{1}, \varepsilon_{2}, \varepsilon_{3}, \varepsilon_{4}\right)^{T}$, that is,

$$
A \varepsilon=\lambda \varepsilon
$$

Taking conjugate transpose $(H)$ on both sides of (3.9), one obtains

$$
\overline{(A \varepsilon)^{T}}=\bar{\lambda} \varepsilon^{H} .
$$

Taking (3.9) multiplied left by $(1 / 2) \varepsilon^{H}$ plus (3.10) multiplied right by $(1 / 2) \varepsilon$, we derive that

$$
\varepsilon^{H}\left(\frac{1}{2} A+\frac{1}{2} A^{H}\right) \varepsilon=\frac{1}{2}(\lambda+\bar{\lambda}) \varepsilon^{H} \varepsilon .
$$

From (3.11), we have

$$
\left(\frac{1}{2}\right)(\lambda+\bar{\lambda})=\frac{\varepsilon^{H}\left((1 / 2) A+(1 / 2) A^{H}\right) \varepsilon}{\varepsilon^{H} \varepsilon} .
$$

By substituting $A$ into (3.12), we can obtain

$$
\frac{1}{2}(\lambda+\bar{\lambda})=\frac{1}{\varepsilon^{H} \varepsilon} \varepsilon^{H}\left[\begin{array}{cccc}
-\alpha & 0 & 0 & 0 \\
0 & -\theta & 0 & 0 \\
0 & 0 & -\gamma & 0 \\
0 & 0 & 0 & -\eta
\end{array}\right] \varepsilon
$$

Since parameters $\alpha, \theta, \gamma$, and $\eta>0, \lambda+\bar{\lambda}<0$, that is, any eigenvalue of matrix $A$ satisfies

$$
|\arg (\lambda)| \geq \frac{\pi}{2}>\frac{q \pi}{2} \quad(q<1)
$$

According to the stability theory of fractional-order systems (Lemma 2.1), error system (3.6) is asymptotically stable, which implies that the inverse projective synchronization between systems (3.1) and (3.2) is achieved. 
It follows from the control law (3.5) that the control law concerns the derive state variables and error variables, but is not related to the response ones. Now, we will propose another control scheme, which is associated with the response state variables and error variables.

Theorem 3.3. For a given constant scaling matrix $\sigma=\operatorname{diag}\left(\sigma_{1}, \sigma_{2}, \sigma_{3}, \sigma_{4}\right)$ and any initial conditions, if parameters $a, b>0$ and $d<0$, then fractional-order hyperchaotic Lorenz system (3.1) and Chen system (3.2) are inverse projective synchronized by the following control law:

$$
\begin{aligned}
u_{1}= & (\alpha-a) y_{1}-\left(\alpha-a \frac{\sigma_{1}}{\sigma_{2}}\right) y_{2}-\left(1-\frac{\sigma_{1}}{\sigma_{4}}\right) y_{4}-\left(c \frac{\sigma_{2}}{\sigma_{1}}+\frac{\sigma_{2}}{\sigma_{1} \sigma_{3}} y_{3}\right) e_{2}+\frac{\sigma_{3}}{\sigma_{1} \sigma_{2}} y_{2} e_{3}, \\
u_{2}= & \left(c \frac{\sigma_{2}}{\sigma_{1}}-\beta\right) y_{1}-(\theta+1) y_{2}+\left(1+\frac{\sigma_{2}}{\sigma_{1} \sigma_{3}}\right) y_{1} y_{3} \\
& +\frac{\sigma_{2}}{\sigma_{1} \sigma_{3}} e_{1} e_{3}-a \frac{\sigma_{1}}{\sigma_{2}} e_{1}+\frac{\sigma_{3}}{\sigma_{1} \sigma_{2}} y_{1} e_{3}-\frac{\sigma_{4}}{\sigma_{2} \sigma_{3}} y_{3} e_{4}, \\
u_{3}= & (r-b) y_{3}-\left(1+\frac{\sigma_{3}}{\sigma_{1} \sigma_{2}}\right) y_{1} y_{2}-\frac{\sigma_{3}}{\sigma_{1} \sigma_{2}} e_{1} e_{2}-\frac{\sigma_{2}}{\sigma_{1} \sigma_{3}} y_{1} e_{2}-\frac{\sigma_{4}}{\sigma_{2} \sigma_{3}} y_{2} e_{4}, \\
u_{4}= & (d-\eta) y_{4}+\left(\frac{\sigma_{4}}{\sigma_{2} \sigma_{3}}-1\right) y_{2} y_{3}-\frac{\sigma_{1}}{\sigma_{4}} e_{1}+\frac{\sigma_{4}}{\sigma_{2} \sigma_{3}} e_{2} e_{3} .
\end{aligned}
$$

Proof. Combining (3.15) with (3.4), the error dynamical system can be described as

$$
\begin{aligned}
& D_{*}^{q} e_{1}=-a e_{1}+\left(a \frac{\sigma_{1}}{\sigma_{2}}-c \frac{\sigma_{2}}{\sigma_{1}}-\frac{\sigma_{2}}{\sigma_{1} \sigma_{3}} y_{3}\right) e_{2}+\frac{\sigma_{3}}{\sigma_{1} \sigma_{2}} y_{2} e_{3}+\frac{\sigma_{1}}{\sigma_{4}} e_{4}, \\
& D_{*}^{q} e_{2}=\left(c \frac{\sigma_{2}}{\sigma_{1}}-a \frac{\sigma_{1}}{\sigma_{2}}+\frac{\sigma_{2}}{\sigma_{1} \sigma_{3}} y_{3}\right) e_{1}-e_{2}+\left(\frac{\sigma_{2}}{\sigma_{1} \sigma_{3}}+\frac{\sigma_{3}}{\sigma_{1} \sigma_{2}}\right) y_{1} e_{3}-\frac{\sigma_{4}}{\sigma_{2} \sigma_{3}} y_{3} e_{4}, \\
& D_{*}^{q} e_{3}=-\frac{\sigma_{3}}{\sigma_{1} \sigma_{2}} y_{2} e_{1}-\left(\frac{\sigma_{2}}{\sigma_{1} \sigma_{3}}+\frac{\sigma_{3}}{\sigma_{1} \sigma_{2}}\right) y_{1} e_{3}-b e_{3}-\frac{\sigma_{4}}{\sigma_{2} \sigma_{3}} y_{2} e_{4}, \\
& D_{*}^{q} e_{4}=-\frac{\sigma_{1}}{\sigma_{4}} e_{1}+\frac{\sigma_{4}}{\sigma_{2} \sigma_{3}} y_{3} e_{2}+\frac{\sigma_{4}}{\sigma_{2} \sigma_{3}} y_{2} e_{3}+d e_{4} .
\end{aligned}
$$

System (3.16) can be rewritten in the following matrix form:

$$
\left[D_{*}^{q} e_{1}, D_{*}^{q} e_{2}, D_{*}^{q} e_{3}, D_{*}^{q} e_{4}\right]^{T}=A\left[e_{1}, e_{2}, e_{3}, e_{4}\right]^{T},
$$

where

$$
A=\left[\begin{array}{cccc}
-a & a \frac{\sigma_{1}}{\sigma_{2}}-c \frac{\sigma_{2}}{\sigma_{1}}-\frac{\sigma_{2}}{\sigma_{1} \sigma_{3}} y_{3} & \frac{\sigma_{3}}{\sigma_{1} \sigma_{2}} y_{2} & \frac{\sigma_{1}}{\sigma_{4}} \\
c \frac{\sigma_{2}}{\sigma_{1}}-a \frac{\sigma_{1}}{\sigma_{2}}+\frac{\sigma_{2}}{\sigma_{1} \sigma_{3}} y_{3} & -1 & \left(\frac{\sigma_{2}}{\sigma_{1} \sigma_{3}}+\frac{\sigma_{3}}{\sigma_{1} \sigma_{2}}\right) y_{1} & -\frac{\sigma_{4}}{\sigma_{2} \sigma_{3}} y_{3} \\
-\frac{\sigma_{3}}{\sigma_{1} \sigma_{2}} y_{2} & -\left(\frac{\sigma_{2}}{\sigma_{1} \sigma_{3}}+\frac{\sigma_{3}}{\sigma_{1} \sigma_{2}}\right) y_{1} & -b & -\frac{\sigma_{4}}{\sigma_{2} \sigma_{3}} y_{2} \\
-\frac{\sigma_{1}}{\sigma_{4}} & \frac{\sigma_{4}}{\sigma_{2} \sigma_{3}} y_{3} & \frac{\sigma_{4}}{\sigma_{2} \sigma_{3}} y_{2} & d
\end{array}\right] .
$$


The following proof process is similar to that of Theorem 3.2, so is omitted here. Finally, we have

$$
\frac{1}{2}(\lambda+\bar{\lambda})=\frac{1}{\varepsilon^{H} \mathcal{\varepsilon}} \varepsilon^{H}\left[\begin{array}{cccc}
-a & 0 & 0 & 0 \\
0 & -1 & 0 & 0 \\
0 & 0 & -b & 0 \\
0 & 0 & 0 & d
\end{array}\right] \varepsilon
$$

Parameters $a, b>0$ and $d<0$, therefore $\lambda+\bar{\lambda}<0$, which means any eigenvalue of matrix $A$ satisfies

$$
|\arg (\lambda)| \geq \frac{\pi}{2}>\frac{q \pi}{2} \quad(q<1)
$$

Based on Lemma 2.1, error system (3.16) is asymptotically stable, which implies the inverse projective synchronization between the drive system (3.1) and the response system (3.2) is achieved under the nonlinear controller (3.15).

If we set the scaling matrix $\sigma=\operatorname{diag}(\sigma, \sigma, \sigma, \sigma)\left(\sigma_{1}=\sigma_{2}=\sigma_{3}=\sigma_{4}=\sigma\right)$, then inverse projective synchronization will be degraded to inverse generalized synchronization. So we have the following Corollaries 3.4 and 3.5 to realize inverse generalized synchronization between the drive system (3.1) and the response system (3.2).

Corollary 3.4. For a given constant scaling matrix $\sigma=\operatorname{diag}(\sigma, \sigma, \sigma, \sigma)$ and any initial conditions, if parameters $\alpha, \theta, \gamma$ and $\eta>0$, then the inverse generalized synchronization between fractional-order hyperchaotic Lorenz system (3.1) and Chen system (3.2) will occur under the following control law:

$$
\begin{aligned}
& u_{1}=(a-\alpha) \sigma x_{1}+(\alpha-a) \sigma x_{2}-\left(\beta+\sigma x_{3}\right) e_{2}+\sigma x_{2} e_{3}, \\
& u_{2}=(\beta-c) \sigma x_{1}+(\theta+1) \sigma x_{2}+\left(\sigma^{2}+\sigma\right) x_{1} x_{3}+\left(e_{3}-\alpha\right) e_{1}-2 \theta e_{2}-\sigma x_{3} e_{4}, \\
& u_{3}=-\left(\sigma^{2}+\sigma\right) x_{1} x_{2}-(\gamma-b) \sigma x_{3}-e_{1} e_{2}+\sigma x_{2} e_{4}, \\
& u_{4}=\left(\sigma-\sigma^{2}\right) x_{2} x_{3}+(\eta-d) \sigma x_{4}-e_{1}-e_{2} e_{3}-2 \eta e_{4} .
\end{aligned}
$$

Corollary 3.5. For a given constant scaling matrix $\sigma=\operatorname{diag}(\sigma, \sigma, \sigma, \sigma)$ and any initial conditions, if parameters $a, b>0$ and $d<0$, then inverse generalized synchronization between fractional-order hyperchaotic Lorenz system (3.1) and Chen system (3.2) are achieved under the following control law:

$$
\begin{aligned}
& u_{1}=(\alpha-a) y_{1}-(\alpha-a) y_{2}-\left(c+\frac{1}{\sigma} y_{3}\right) e_{2}+\frac{1}{\sigma} y_{2} e_{3} \\
& u_{2}=(c-\beta) y_{1}-(\theta+1) y_{2}+\left(1+\frac{1}{\sigma}\right) y_{1} y_{3}+\frac{1}{\sigma} e_{1} e_{3}-a e_{1}+\frac{1}{\sigma} y_{1} e_{3}-\frac{1}{\sigma} y_{3} e_{4}
\end{aligned}
$$




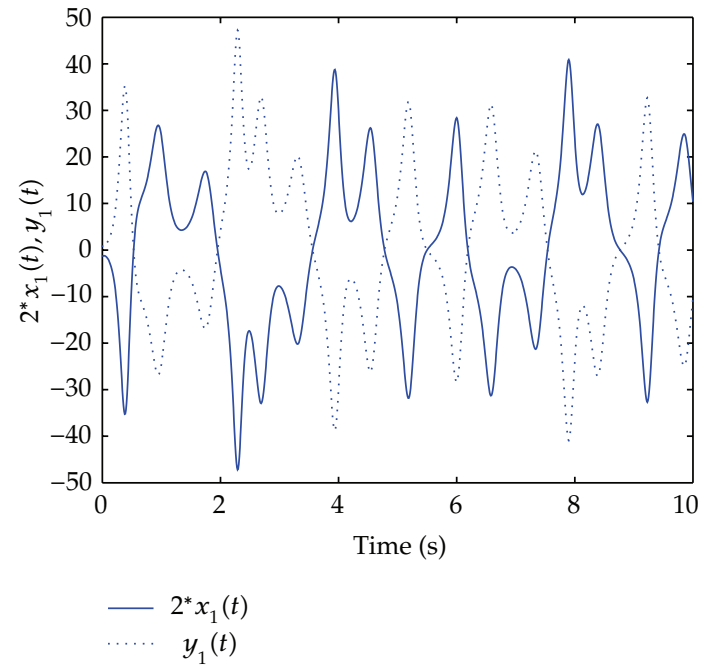

(a)

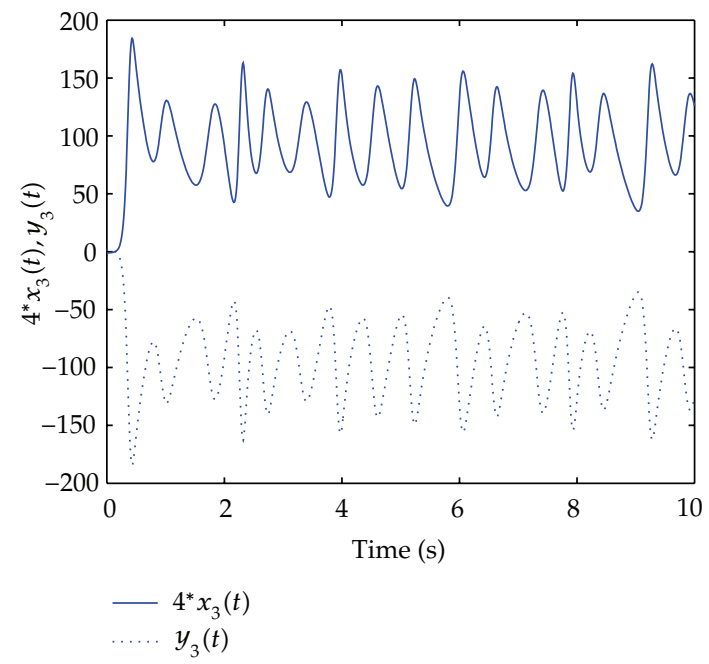

(c)

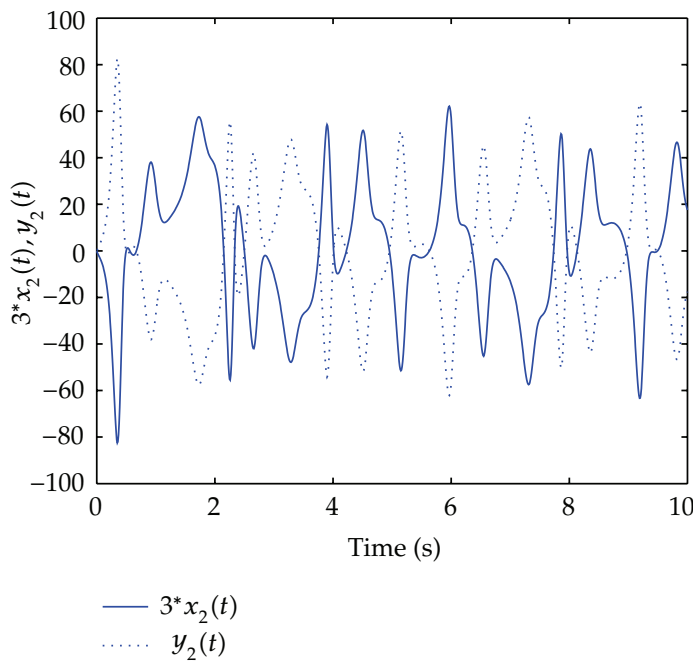

(b)

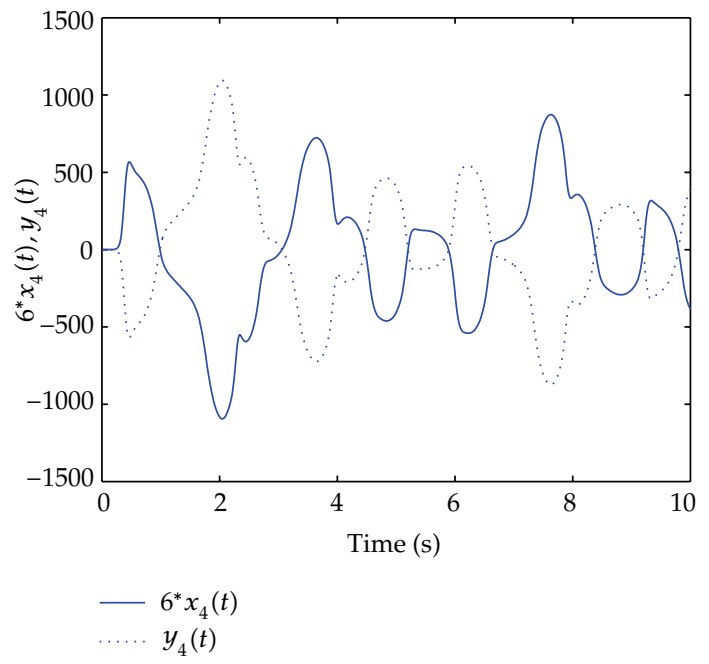

(d)

Figure 4: The state synchronization trajectories of the master system (3.1) and slave system (3.2) under control law (3.5).

$$
\begin{aligned}
& u_{3}=(r-b) y_{3}-\left(1+\frac{1}{\sigma}\right) y_{1} y_{2}-\frac{1}{\sigma} e_{1} e_{2}-\frac{1}{\sigma} y_{1} e_{2}-\frac{1}{\sigma} y_{2} e_{4}, \\
& u_{4}=(d-\eta) y_{4}+\left(\frac{1}{\sigma}-1\right) y_{2} y_{3}-e_{1}+\frac{1}{\sigma} e_{2} e_{3} .
\end{aligned}
$$

Further, we take constant scaling matrix $\sigma$ as $\sigma=\operatorname{diag}(1,1,1,1)\left(\sigma_{1}=\sigma_{2}=\sigma_{3}=\sigma_{4}=1\right)$, antisynchronization between the derive system (3.1) and the response system (3.2) will occur by the following Corollaries 3.6 and 3.7 . 


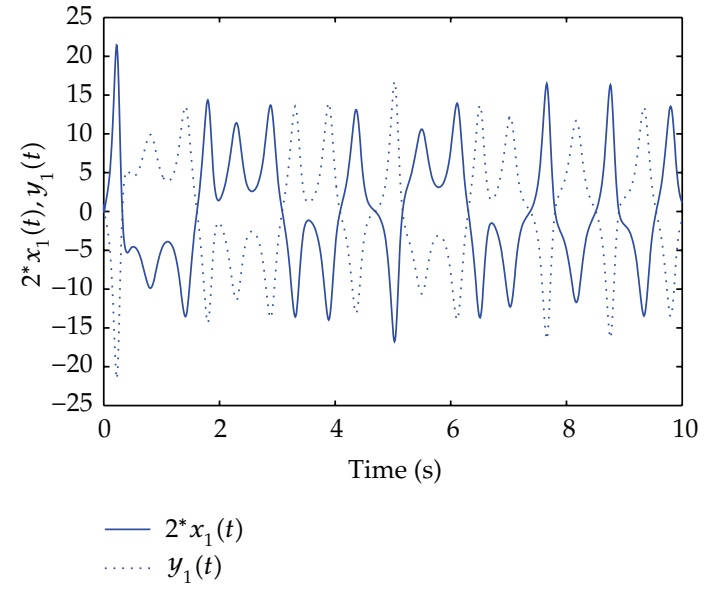

(a)

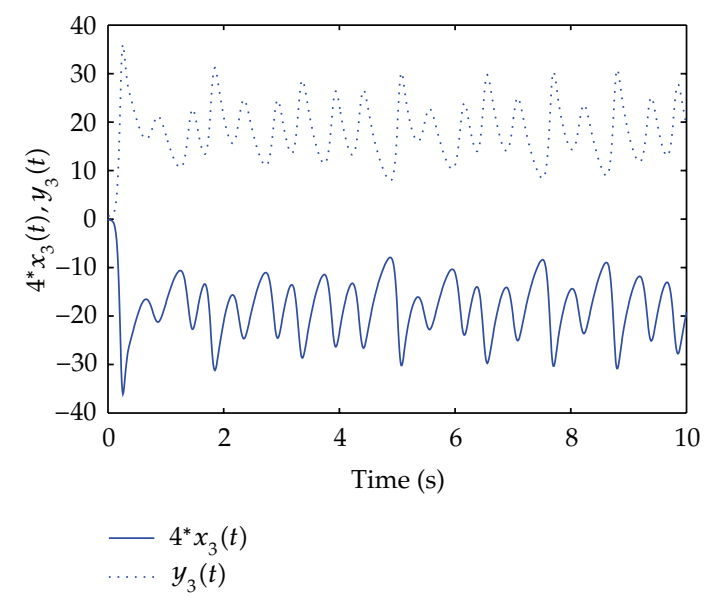

(c)

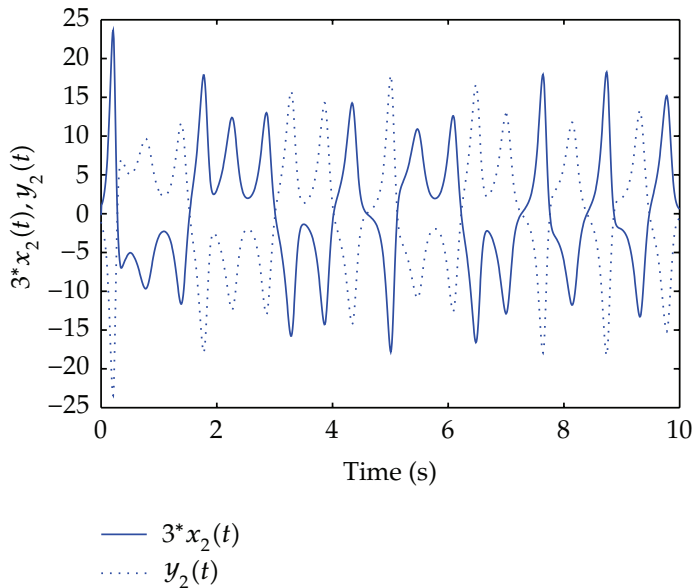

(b)

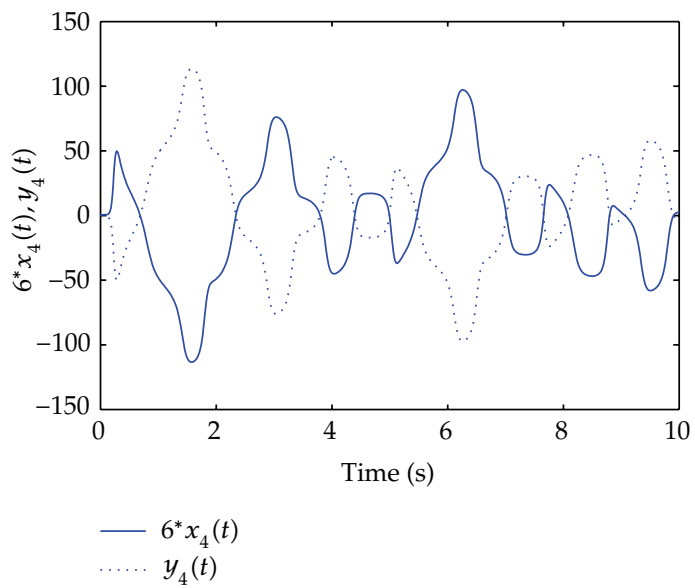

(d)

Figure 5: The state synchronization trajectories of the master system (3.1) and slave system (3.2) under control law (3.15).

Corollary 3.6. For any initial conditions, if parameters $\alpha, \theta, \gamma$, and $\eta>0$, then antisynchronization between fractional-order hyperchaotic Lorenz system (3.1) and Chen system (3.2) will occur under the following control law:

$$
\begin{aligned}
& u_{1}=(a-\alpha) x_{1}+(\alpha-a) x_{2}-\left(\beta+x_{3}\right) e_{2}+x_{2} e_{3}, \\
& u_{2}=(\beta-c) x_{1}+(\theta+1) x_{2}+2 x_{1} x_{3}+\left(e_{3}-\alpha\right) e_{1}-2 \theta e_{2}+x_{3} e_{4}, \\
& u_{3}=-2 x_{1} x_{2}-(\gamma-b) x_{3}-e_{1} e_{2}+x_{2} e_{4}, \\
& u_{4}=(\eta-d) x_{4}-e_{1}-e_{2} e_{3}-2 \eta e_{4} .
\end{aligned}
$$




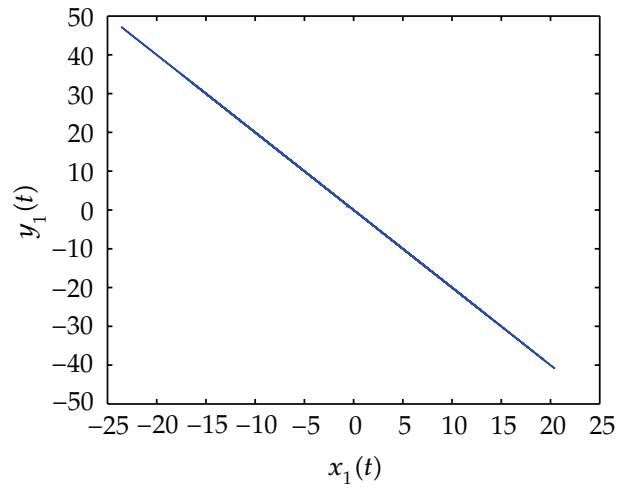

(a)

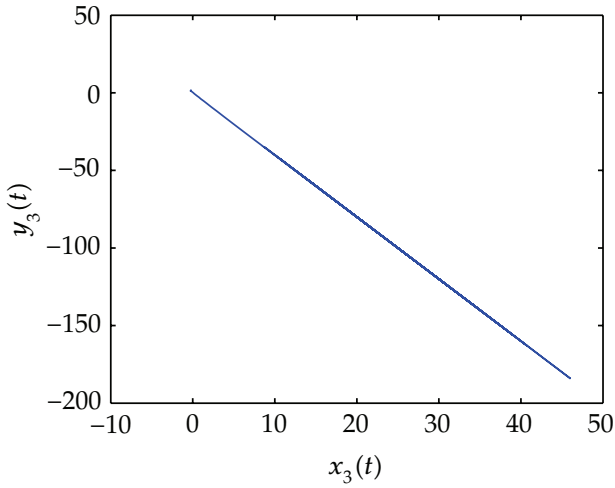

(c)

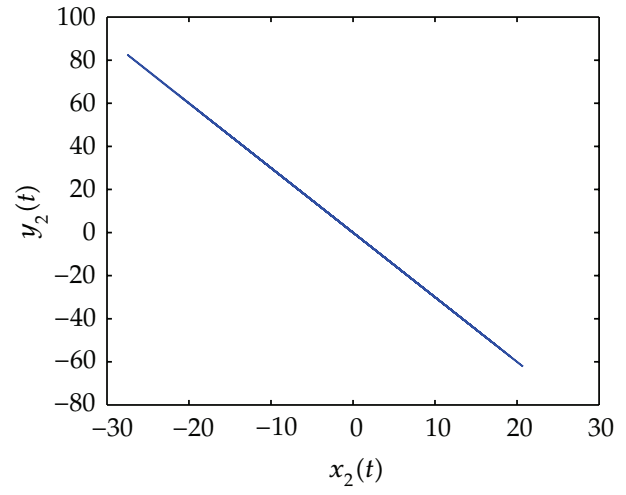

(b)

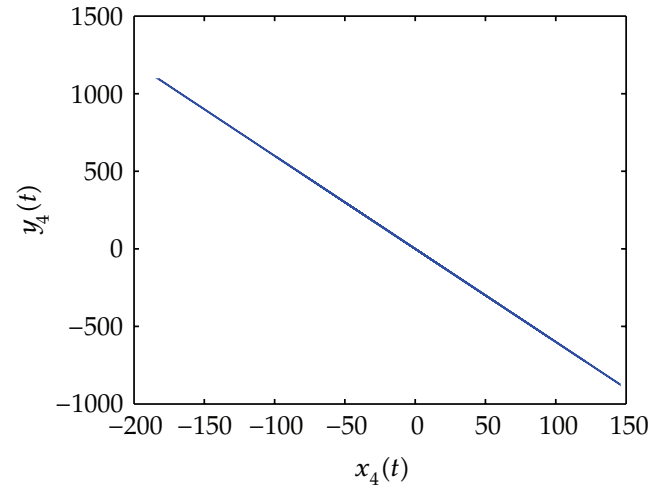

(d)

Figure 6: Signals after synchronization under control law (3.5).

Corollary 3.7. For any initial conditions, if the parameters $a, b>0$ and $d<0$, then fractional-order hyperchaotic Lorenz system (3.1) and Chen system (3.2) are inverse generalized synchronized under the following control law:

$$
\begin{aligned}
& u_{1}=(\alpha-a) y_{1}-(\alpha-a) y_{2}-\left(c+y_{3}\right) e_{2}+y_{2} e_{3}, \\
& u_{2}=(c-\beta) y_{1}-(\theta+1) y_{2}+2 y_{1} y_{3}+e_{1} e_{3}-a e_{1}+y_{1} e_{3}-y_{3} e_{4} \\
& u_{3}=(r-b) y_{3}-2 y_{1} y_{2}-e_{1} e_{2}-y_{1} e_{2}-y_{2} e_{4} \\
& u_{4}=(d-\eta) y_{4}-e_{1}+e_{2} e_{3}
\end{aligned}
$$

Similarly, scaling factor matrices are selected as $\sigma=\operatorname{diag}(-\sigma,-\sigma,-\sigma,-\sigma)$ and $\sigma=$ $\operatorname{diag}(-1,-1,-1,-1)$; it is easy to obtain the corresponding corollaries to achieve generalized synchronization and complete synchronization, respectively. We omit here.

\section{Numerical Simulations}

In this section, to verify and demonstrate the effectiveness of the proposed controller laws, we discuss the simulation results of inverse projective synchronization between fractional-order 


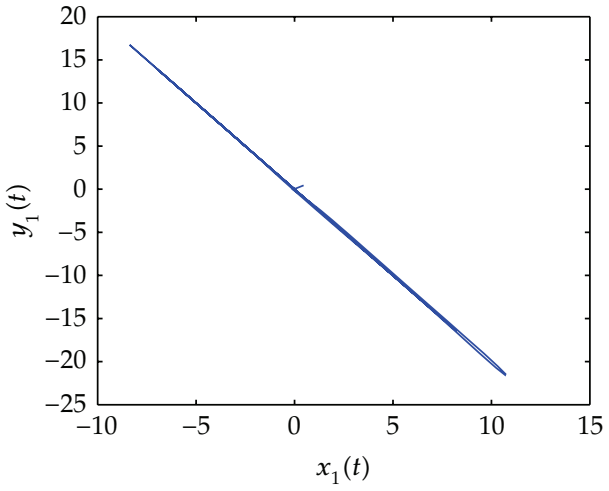

(a)

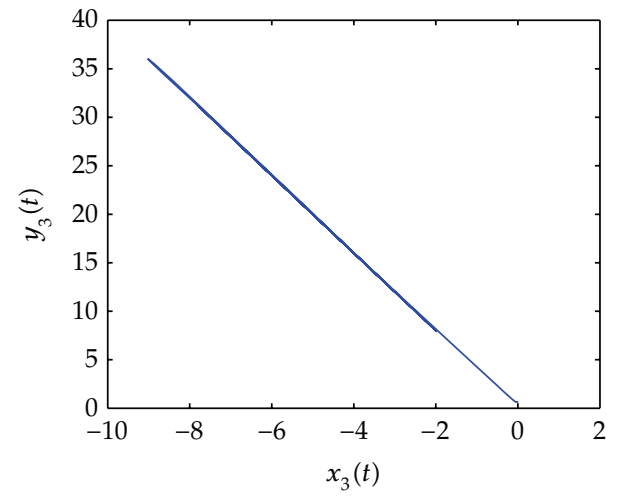

(c)

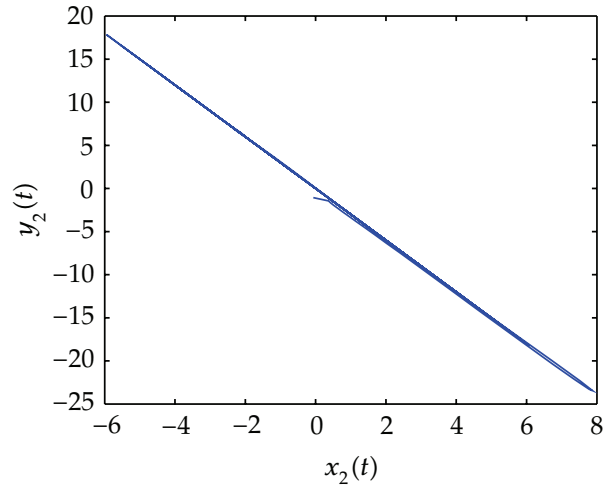

(b)

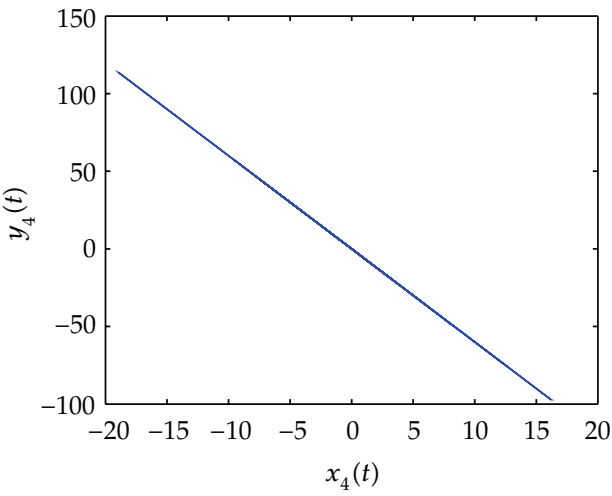

(d)

Figure 7: Signals after synchronization under control law (3.15).

hyperchaotic Lorenz system and fractional-order hyperchaotic Chen system. The simulation is performed using Matlab software.

For this numerical simulation, the parameters are chosen to be $\alpha=35, \beta=7, \theta=12$, $\gamma=3, \eta=0.5, a=10, b=8 / 3, c=28$, and $d=-1$, which all satisfy the conditions $\alpha, \theta, \gamma, \eta>0$ and $a, b>0, d<0$ in Theorems 3.2 and 3.3, respectively. The initial values of the drive and response systems are $\left(x_{1}(0), x_{2}(0), x_{3}(0), x_{4}(0)\right)=(-0.8,0.2,-0.3,0.1)$ and $\left(y_{1}(0), y_{2}(0), y_{3}(0), y_{4}(0)\right)=(1.2,-1.2,0.7-0.3)$, respectively. Without loss of generality, the scaling matrix $\sigma$ is chosen as $\operatorname{diag}(2,3,4,6)$ and $q=0.98$. Based on Theorems 3.2 and 3.3, under the control laws (3.5) and (3.15), the state variables during the synchronizing process between the fractional-order hyperchaotic Lorenz system and fractional-order hyperchaotic Chen system are shown in Figures 4 and 5, respectively. Figures 6 and 7 show the signals after synchronization under the control laws (3.5) and (3.15), respectively. It is obvious that the state vectors of the systems are synchronized in the same direction all the time from the simulations. Define $e(t)=\sqrt{e_{1}(t)^{2}+e_{2}(t)^{2}+e_{3}(t)^{2}+e_{4}(t)^{2}}$. Figures $8(\mathrm{a})$ and $8(\mathrm{~b})$ display the synchronization errors between systems (3.1) and (3.2) under the control laws (3.5) and (3.15), respectively. 


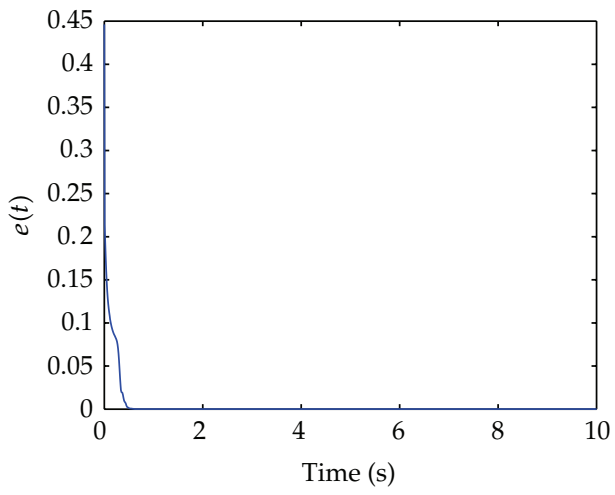

(a)

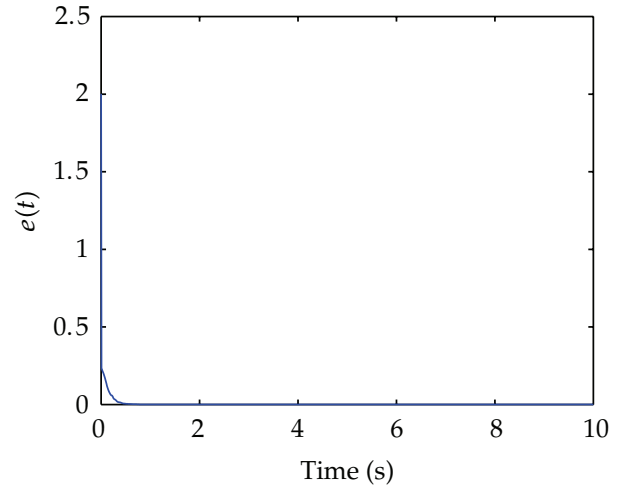

(b)

Figure 8: Error states of the drive system (3.1) and the response system (control law (3.5): (a); control law $(3.15):(b))$.

\section{Conclusions}

In this paper, based on the stability theory of fractional-order differential equations and Lyapunov equations for fractional-order systems, two criteria are provided to ensure the inverse projective synchronization between fractional-order hyperchaotic Lorenz system and fractional-order hyperchaotic Chen system. There are few papers about the inverse projective synchronization of fractional-order hyperchaotic system, which often exists in real systems. The feasibility and effectiveness of proposed schemes have been validated by computer simulation. It should be noted that complete synchronization, antisynchronization, and general projective synchronization are the special cases of inverse projective synchronization. Therefore, the results of this paper are more applicable and representative. Meanwhile, results could be extended to other fractional-order hyperchaotic system, such as fractional-order hyperchaotic Lü system, and fractional-order hyperchaotic Rössler system.

\section{Acknowledgments}

This work is supported by the National Natural Science Foundation of China (no. 60974090), the Fundamental Research Funds for the Central Universities (no. CDJXS11172237; no. CDJRC10170005; no. CDJZR 11170005), the Specialized Research Fund for the Doctoral Program of Higher Education of China (no. 20093401120001; no. 102063720090013), the Natural Science Foundation of Anhui Province (no. 11040606M12), and the Natural Science Foundation of Anhui Education Bureau (no. KJ2010A035).

\section{References}

[1] R. C. Koeller, "Polynomial operators, Stieltjes convolution, and fractional calculus in hereditary mechanics," Acta Mechanica, vol. 58, no. 3-4, pp. 251-264, 1986.

[2] R. C. Koeller, "Applications of fractional calculus to the theory of viscoelasticity," Journal of Applied Mechanics, vol. 51, no. 2, pp. 299-307, 1984.

[3] O. Heaviside, Electromagnetic theory, Chelsea, New York, NY, USA, 1971.

[4] I. Podlubny, "Fractional-order systems and $\mathrm{PI}^{\curlywedge} D^{\mu}$-controllers," IEEE Transactions on Automatic Control, vol. 44, no. 1, pp. 208-214, 1999. 
[5] F. B. M. Duarte and J. A. T. Machado, "Chaotic phenomena and fractional-order dynamics in the trajectory control of redundant manipulators," Nonlinear Dynamics, vol. 29, no. 1-4, pp. 315-342, 2002.

[6] C. P. Li, W. H. Deng, and D. Xu, "Chaos synchronization of the Chua system with a fractional order," Physica A, vol. 360, no. 2, pp. 171-185, 2006.

[7] C. Li and G. Chen, "Chaos and hyperchaos in the fractional-order Rössler equations," Physica A, vol. 341, no. 1-4, pp. 55-61, 2004.

[8] C. Li and G. Peng, "Chaos in Chen's system with a fractional order," Chaos, Solitons E Fractals, vol. 22, no. 2, pp. 443-450, 2004.

[9] L.-J. Sheu, H.-K. Chen, J.-H. Chen et al., "Chaos in the Newton-Leipnik system with fractional order," Chaos, Solitons \& Fractals, vol. 36, no. 1, pp. 98-103, 2008.

[10] W. H. Deng and C. P. Li, “Chaos synchronization of the fractional Lü system,” Physica A, vol. 353, no. 1-4, pp. 61-72, 2005.

[11] X. Gao and J. B. Yu, "Chaos in the fractional order periodically forced complex Duffing's oscillators," Chaos, Solitons \& Fractals, vol. 24, no. 4, pp. 1097-1104, 2005.

[12] X. Wu and Y. Lu, "Generalized projective synchronization of the fractional-order Chen hyperchaotic system," Nonlinear Dynamics, vol. 57, no. 1-2, pp. 25-35, 2009.

[13] T. Wang and X. Wang, "Generalized synchronization of fractional order hyperchaotic lorenz system," Modern Physics Letters B, vol. 23, no. 17, pp. 2167-2178, 2009.

[14] Y. G. Yu and H.-X. Li, "The synchronization of fractional-order Rössler hyperchaotic systems," Physica A, vol. 387, no. 5-6, pp. 1393-1403, 2008.

[15] L. M. Pecora and T. L. Carroll, "Synchronization in chaotic systems," Physical Review Letters, vol. 64, no. 8, pp. 821-824, 1990.

[16] G. R. Chen and X. Dong, From Chaos to Order-Perspectives, Methodologies and Applications, World Scientific, Singapore, 1998.

[17] S. K. Dana, P. K. Roy, and J. Kurths, Complex Dynamics in Physiological Systems, from Heart to Brain, Springer, New York, NY, USA, 2009.

[18] C. Li and X. F. Liao, "Complete and lag synchronization of hyperchaotic systems using small impulses," Chaos, Solitons E Fractals, vol. 22, no. 4, pp. 857-867, 2004.

[19] G. H. Li, "Projective lag synchronization in chaotic systems," Chaos, Solitons E Fractals, vol. 41, no. 5, pp. 2630-2634, 2009.

[20] X. D. Zhang, P. D. Zhao, and A. H. Li, "Construction of a new fractional chaotic system and generalized synchronization," Communications in Theoretical Physics, vol. 53, no. 6, pp. 1105-1110, 2010.

[21] H. Jiang and Q. Bi, "Impulsive synchronization of networked nonlinear dynamical systems," Physics Letters A, vol. 374, no. 27, pp. 2723-2729, 2010.

[22] X. F. Lang, Q. S. Lu, and K. Jurge, "Phase synchronization in noise-driven bursting neurons," Physical Review E, vol. 82, no. 2, pp. 1-6, 2010.

[23] I. Wedekind and U. Parlitz, "Experimental observation of synchronization and anti-synchronization of chaotic low-frequency-fluctuations in external cavity semiconductor lasers," International Journal of Bifurcation and Chaos in Applied Sciences and Engineering, vol. 11, no. 4, pp. 1141-1147, 2001.

[24] M. K. Chil, S. H. Rim, W. H. Kye, J. W. Ryu, and Y. J. Park, "Anti-synchronization of chaotic oscillators," Physics Letters A, vol. 320, no. 1, pp. 39-46, 2003.

[25] J. Hu, S. Chen, and L. Chen, "Adaptive control for anti-synchronization of Chua's chaotic system," Physics Letters A, vol. 339, no. 6, pp. 455-460, 2005.

[26] G. H. Li and S. P. Zhou, "Anti-synchronization in different chaotic systems," Chaos, Solitons \& Fractals, vol. 32, no. 2, pp. 516-520, 2007.

[27] L. Pan, W. Zhou, J. Fang, and D. Li, "A novel active pinning control for synchronization and antisynchronization of new uncertain unified chaotic systems," Nonlinear Dynamics, vol. 62, no. 1-2, pp. 417-425, 2010.

[28] J. M. González-Miranda, "Synchronization of symmetric chaotic systems," Physical Review E, vol. 53, no. 6, pp. 5656-5669, 1996.

[29] R. Mainieri and J. Rehacek, "Projective synchronization in three-dimensional chaotic systems," Physical Review Letters, vol. 82, no. 15, pp. 3042-3045, 1999.

[30] G.-H. Li, "Modified projective synchronization of chaotic system," Chaos, Solitons E Fractals, vol. 32, no. 5, pp. 1786-1790, 2007.

[31] Q. Jia, "Projective synchronization of a new hyperchaotic Lorenz system," Physics Letters A, vol. 370, no. 1 , pp. $40-45,2007$.

[32] M. F. Hu and Z. Y. Xu, "Adaptive feedback controller for projective synchronization," Nonlinear Analysis: Real World Applications, vol. 9, no. 3, pp. 1253-1260, 2008. 
[33] D. Ghosh, "Projective synchronization in multiple modulated time-delayed systems with adaptive scaling factor," Nonlinear Dynamics, vol. 62, no. 4, pp. 751-759, 2010.

[34] S. Shao, "Controlling general projective synchronization of fractional order Rossler systems," Chaos, Solitons \& Fractals, vol. 39, no. 4, pp. 1572-1577, 2009.

[35] G. Peng and Y. Jiang, "Generalized projective synchronization of a class of fractional-order chaotic systems via a scalar transmitted signal," Physics Letters A, vol. 372, no. 22, pp. 3963-3970, 2008.

[36] X. Wu and H. Wang, "A new chaotic system with fractional order and its projective synchronization," Nonlinear Dynamics, vol. 61, no. 3, pp. 407-417, 2010.

[37] S. Wang, Y. Yu, and M. Diao, "Hybrid projective synchronization of chaotic fractional order systems with different dimensions," Physica A, vol. 389, no. 21, pp. 4981-4988, 2010.

[38] L. Pan, W. Zhou, J. Fang, and D. Li, "Synchronization and anti-synchronization of new uncertain fractional-order modified unified chaotic systems via novel active pinning control," Communications in Nonlinear Science and Numerical Simulation, vol. 15, no. 12, pp. 3754-3760, 2010.

[39] H. M. Deng, T. H. Li, Q. Wang, and H. Li, "A fractional-order hyperchaotic system and its synchronization," Chaos, Solitons \& Fractals, vol. 41, no. 2, pp. 962-969, 2009.

[40] N. Sun, H. G. Zhang, Z. L. Wang, and M. Dong, "Anti-synchronization of fractional-order hyperchaotic systems via sliding mode controller," in Proceedings of the 29th Chinese Control Conference (CCC '10), pp. 755-759, July 2010.

[41] H. W. Songa, X. R. Lin, H. Yang, R. Zhen, Y. Chie, and X. Feng, "Full state hybrid projective synchronization in fractional Chen-Lee hyperchaotic system," in Proceedings of the International Conference on Communications, Circuits and Systems (ICCCAS '10), pp. 777-780, July 2010. 


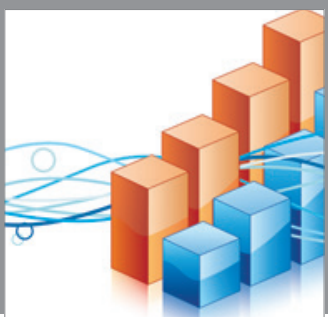

Advances in

Operations Research

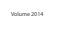

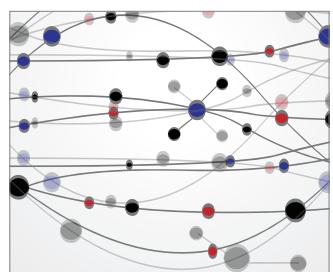

\section{The Scientific} World Journal
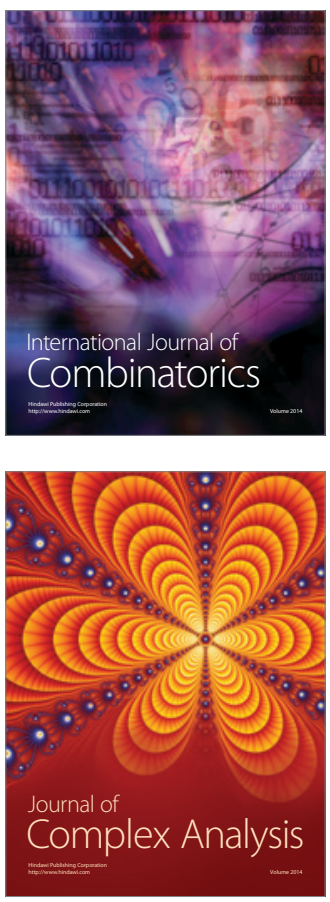

International Journal of

Mathematics and

Mathematical

Sciences
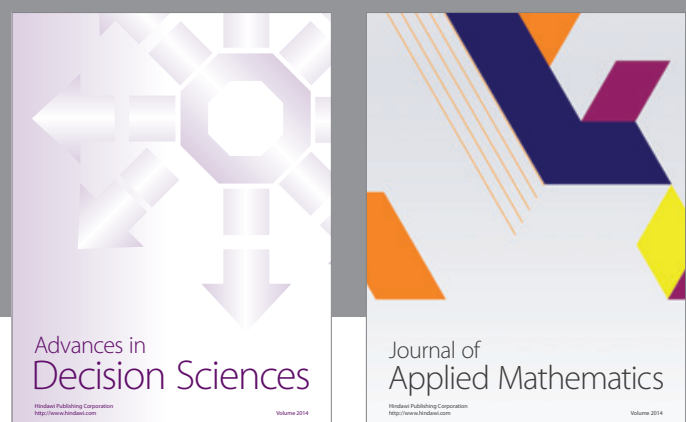

Journal of

Applied Mathematics
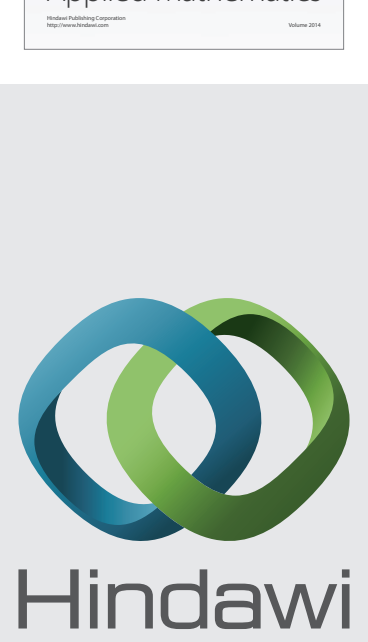

Submit your manuscripts at http://www.hindawi.com
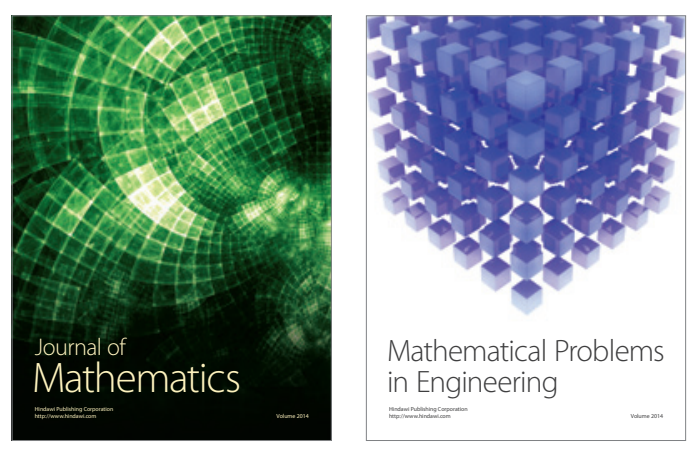

Mathematical Problems in Engineering
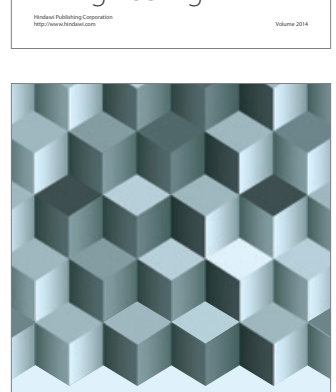

Journal of

Function Spaces
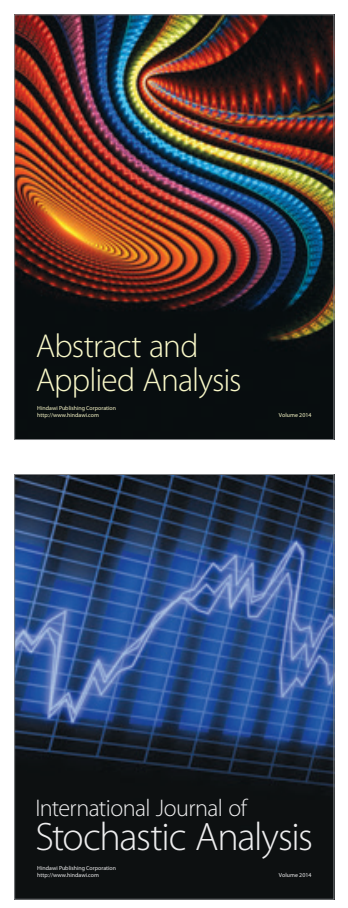

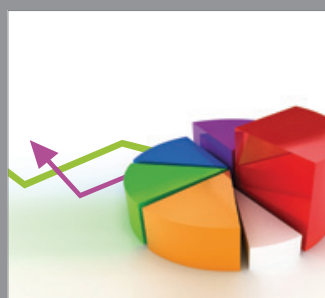

ournal of

Probability and Statistics

Promensencen
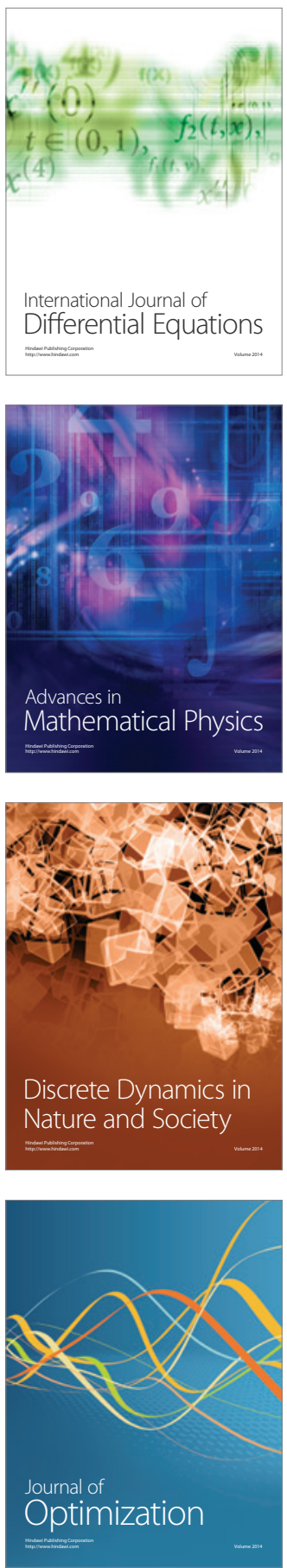